\title{
EchoGéo
}

15 | 2011

La Belgique, État prospère en décomposition au cœur de l'Europe du nord-ouest

\section{Les études postcoloniales. Un carnaval académique} Compte rendu de lecture

Philippe Gervais-Lambony

\section{OpenEdition \\ Journals}

Édition électronique

URL : https://journals.openedition.org/echogeo/12310

DOI : 10.4000/echogeo.12310

ISSN : 1963-1197

Éditeur

Pôle de recherche pour l'organisation et la diffusion de l'information géographique (CNRS UMR 8586)

\section{Référence électronique}

Philippe Gervais-Lambony, "Les études postcoloniales. Un carnaval académique », EchoGéo [En ligne], 15 | 2011, mis en ligne le 11 avril 2011, consulté le 03 août 2021. URL : http://journals.openedition.org/ echogeo/12310 ; DOI : https://doi.org/10.4000/echogeo.12310

\section{Ce document a été généré automatiquement le 3 août 2021}

EchoGéo est mis à disposition selon les termes de la licence Creative Commons Attribution - Pas d'Utilisation Commerciale - Pas de Modification 4.0 International (CC BY-NC-ND) 


\title{
Les études postcoloniales. Un carnaval académique
}

\author{
Compte rendu de lecture
}

Philippe Gervais-Lambony

1 Il faut saluer la création d'une nouvelle et prometteuse collection aux éditions Karthala. Appelée Disputatio, elle se propose de prendre part, à travers des ouvrages brefs, à des débats scientifiques sans rechigner à la polémique. Il est probable que ceci devrait être plus développé dans les sciences sociales françaises. L'ouvrage de JeanFrançois Bayart exemplifie magistralement l'intérêt de l'exercice. Ce petit livre aussi efficace que polémique (comme son sous-titre l'indique assez) se propose d'aborder «bille en tête » la question du "postcolonial ». Ceci concerne l'ensemble des sciences sociales puisque ce dont il est question est bien l'hypothèse d'une approche nouvelle dans leurs divers champs, et répond à la mise en cause des approches dites «classiques »; notamment la géographie est concernée puisque, comme les autres disciplines académiques, elle est interrogée par des auteurs qui se revendiquent d'une approche postcoloniale ou proposent au moins d'en mobiliser des éléments.

2 Jean-François Bayart est ici critique et réagit à la fois à une mode qui consiste à appliquer à tout va le terme postcolonial à des situations très diverses et à une critique qu'il juge injustement adressée aux sciences sociales françaises, celle d'avoir manqué le «tournant " postcolonial. C'est une défense en règle couplée à une attaque qui l'est tout autant. On conseillera cet ouvrage à tous ceux qui s'intéressent à cette question, tout particulièrement à ceux qui travaillent sur des pays du Sud, mais pas seulement bien sûr, loin de là, puisque ceci concerne aussi aujourd'hui la manière de comprendre les anciennes puissances coloniales (comme le souligne Achille Mbembe dans un ouvrage lui aussi publié en 2010 : Sortir de la Grande Nuit, Essai sur l'Afrique décolonisée, Paris, La Découverte; d'une certaine manière, indirecte, les deux livres peuvent d'ailleurs être lus comme un échange entre les deux auteurs). Il ne me semble pas qu'il existait d'ouvrage sur la question aussi complet en français et appuyé sur une aussi vaste bibliographie. C'est dire que pour critique qu'il soit, le livre de Jean-François Bayart est aussi une introduction aux études postcoloniales puisqu'on y trouve dans un 
appareil de notes et une bibliographie analytique l'essentiel des références indispensables.

3 De quoi est-il question? De rien de moins que de l'hypothèse d'une incompréhension du monde par les sciences sociales produites selon des normes «occidentales", de la proposition d'une réécriture de l'histoire des sociétés contemporaines à une nouvelle lumière, du projet de re-définition de la relation à l'Autre (comprise aussi comme servant à la définition du "nous ", d'où le regard porté aussi sur les "anciens " pays colonisateurs). Tout ceci conduisant aussi à une mise en cause de l'objectivité de la science et au projet aussi d'en modifier les méthodes de recherche et d'exposition des résultats.

4 L'ouvrage de Jean-François Bayart semble construit en trois grands chapitres, qui suivent une introduction très claire sur les différentes formes de postcolonial studies, mais il est en fait plutôt d'une seule traite, comme un jet. Le premier argument de l'auteur: on ne peut parler d'un courant postcolonial (dont on n'arrive d'ailleurs pas clairement même à décider s'il faut l'orthographier avec ou sans tiret...) puisqu'il s'agit d'une "rivière aux multiples affluents». Il convient de distinguer la critique de l'Orientalisme, initiée par Edward Said à la fin des années 1970 et prolongée par la critique de l'africanisme, la critique littéraire (venant d'auteurs Indiens, Caribéens et Africains), la critique philosophique issue de la french theory et du postmodernisme, les réflexions sur l'histoire de la traite et de l'Atlantique noir. Au-delà des convergences entre ces courants (et leur capacité à établir le lien entre le colonialisme et les autres formes de domination est une qualité reconnue par l'auteur aux postcoloniales studies), Jean-François Bayart interroge l'ambiguïté de travaux qui oscillent entre analyse scientifique et engagement sociétal (dont les liens avec des mouvements sociaux de défense de minorités est clair, et d'ailleurs en général pleinement assumé ; sur ce point il y a peut-être un malentendu car la distinction entre les deux ordres est précisément ce qui est mis en cause par les auteurs ici critiqués). Les sciences sociales françaises ont-elles manqué ces différents coches ? C'est ce dont on les accuse, injustement, selon Jean-François Bayart. Injustement pour différentes raisons. Premièrement, et les pages sur la situation des études africanistes françaises sont d'une remarquable lucidité, parce que c'est la politique scientifique française qui est en cause et son manque de moyens et plus encore peut-être la politique de relation avec les universités africaines laissées dans un extraordinaire dénuement alors même qu'une " politique imbécile en matière de visas (...) a rendu de facto impossibles les échanges scientifiques avec les pays du Sud depuis le milieu des années 1980 »(p. 35)... Deuxièmement parce que les approches proposées par les auteurs postcoloniaux sont en fait présentes dans les sciences sociales françaises, et depuis longtemps, d'où le titre du deuxième chapitre : " on a déjà donné ». Pourquoi ? Parce que les références centrales des postcolonial studies sont en langue françaises et débattues depuis longtemps, au premier chef les textes de Frantz Fanon, mais aussi Césaire et Senghor. Troisièmement parce que les auteurs français ont produit nombre de travaux qui déconstruisent en effet l'histoire coloniale, mais avec moins de fureur de bruit que les postcoloniaux, avec, selon Jean-François Bayart, modestie et surtout remise en contexte des différentes situations coloniales dans une tradition académique qu'il faut défendre.

5 C'est essentiellement sur cette question que se poursuit le propos du livre pour souligner un certains nombre de faiblesses du courant discuté. Laissons de côté peutêtre les aspects les plus personnels (à savoir que la plupart des postcoloniaux parlent 
en fait depuis une position diasporique depuis un pays du Nord, position confortable souvent qui les rendrait illégitime pour affirmer parler depuis «le Sud»), mais voyons le fond et deux critiques principales. Tout d'abord le caractère essentialisant et réificateur des études postcoloniales : les pays du nord seraient essentialisés et donc critiqués sans une analyse tenant compte suffisament de leur diversité (aussi interne), la colonialisme serait quant à lui réifié, et du coup on en serait conduit dans une impasse qui rendrait impossible la compréhesion des dynamiques, des évolutions historiques (de même les auteurs postcoloniaux sont ici accusés de simplification, au sens de mise sur le même plan de réalités historiques fondamentalement différentes, mais certains "postcoloniaux" l'ont souligné depuis longtemps tel Stuart Hall: on n'est pas postcolonial de la même manière au Nigeria et en Australie... On notera qu'Hall n'est pas cité dans l'ouvrage, il pourrait être utile pourtant en tant qu'auteur majeur des cultural studies puis des postcolonial studies qui a été un des premiers à dénoncer les dérives de l'une et l'autre quand le culturel est devenu objet unique ce qui a conduit à la dépolitisation d'un courant de pensée né pourtant d'une volonté d'engagement politique en débat avec les approches marxistes jugées trop économistes et eurocentrées (voir Hall Stuart, Identités et cultures, politiques des cultural studies, Paris, Editions Amsterdam, 2007). C'est précisément l'autre critique majeure portée par JeanFrançois Bayart, le lien entre le postcolonial et le «tournant culturel»: «le grand reproche que l'on peut adresser aux postcolonial studies tient à leur adhésion aux formes les plus extrêmes du cultural turn des années 1980-1990» (page 44). Alors les études postcoloniales auraient dérivé vers une analyse de discours, vers une extrapolation à partir de ces discours sans véritable travail de terrain, d'archive, sans attention non plus à la diversité historique des situations réelles (par exemple la différence entre les colonies de peuplement, les colonies esclavagistes et les autres) ni à la sociologie des groupes. L'exemple qui est mobilisé à plusieurs reprises par Jean-François Bayart pour souligner les dangers d'un tel glissement est celui de l'ethnicisation de la question sociale des banlieues françaises et l'occultation des questions de classe.

6 Finalement, l'auteur veut proposer une voie différente de compréhension du postcolonial, une voie qui en accepte l'historicité et la diversité, avec un objectif à la fois scientifique et mobilisateur. Insistons sur ce deuxième point: rendre au fait colonial son historicité est bel et bien une question politique puisque c'est aussi la condition pour en sortir. L'argument est ici que si l'on s'en tient à une lecture univoque et mécanique du fait colonial on se trouve face à une situation postcoloniale elle aussi a-historique et en conséquence inexpugnable. Au bout du compte ne peut-on en effet reprocher aux auteurs postcoloniaux de participer «à la reproduction de l'hégémonie coloniale » (page 66) parce qu'ils mettent en avant le caractère surdéterminant du colonial et la continuité entre colonial et postcolonial ? Il y a là indiscutablement un débat majeur et urgent.

Un ouvrage de débat donc, et tout aussi utile que remarquablement argumenté. Une analyse géographique aurait certes ajouté des éléments sur l'importance de la prise en compte des diversités spatiales autant qu'historiques. Mais ce n'est sans doute pas l'essentiel qui est plutôt à chercher dans une interrogation sur le positionnement de la géographie (et notamment française) dans ce débat sur le postcolonial (puisque JeanFrançois Bayart l'aborde bien sûr d'abord du point de vue d'un politologue). La mise en cause des approches «traditionnelles » de la géographie par les études postcoloniales a apporté beaucoup à mon sens et est loin d'avoir fini d'avoir des effets. C'est d'ailleurs une critique que l'on pourrait faire à Jean-François Bayart mais qui est liée au style de 
l'ouvrage : n'aurait-il pas été légitime de rassembler à un moment donné les apports des études postcoloniales. Ils ne sont pas niés, mais ils sont disséminés et semblent toujours l'être avec réticence. « La bibliothèque postcoloniale, pour le meilleur est pour le pire... » (page 99), titre la bibliographie, alors pourquoi ne pas faire plus nettement la part de l'un et de l'autre? La mise en relation des différentes formes d'oppression culturelle, la déconstruction de processus de domination, sont deux éléments importants et soulignés (discrètement) par Jean-François Bayart. La mise en cause de l'objectivité de la recherche, le questionnement sur la positionnalité de l'auteur d'un texte scientifique, la réflexion sur les méthodes de la recherche,l'effort pour adopter la perspective de l'autre (qui est en l'occurrence souvent l'objet de la recherche) me semblent aussi des points essentiels mais négligés ici. Tout comme les auteurs se revendiquant d'approches post-modernes, les postcoloniaux ont la grande qualité de faire douter les sciences sociales, et je suis convaincu que le doute nous fait progresser plus que les certitudes. En contrepoint à la lecture de l'ouvrage de Jean-François Bayart je recommanderais donc volontiers la lecture du livre très éclairant de Joanne P. Sharp Geographies of Postcolonialism, Spaces of Power and representation (SAGE, 2009) qui propose une géographie postcoloniale prometteuse. De la même manière on peut conseiller la lecture de Jennifer Robinson (Ordinary Cities, Between modernity and Development, Routledge, Londres, 2006) qui promeut des études urbaines postcoloniales qui reposeraient sur l'acceptation d'un regard ouvert sur le monde, sans rejet dans la marginalité de la non-modernité ou du seul besoin de " développement » de la majorité des citadins de la planète. Ces ouvrages sont des approches de géographes et disent à les apports possibles des études postcoloniales pour la discipline, apports déjà manifeste dans le monde scientifique anglophone et de plus en plus chez une jeune génération de géographes français : proposer une perspective différente, bousculer les lignes, sortir des catégorisations dominantes et en général stérilisantes. La plus forte d'entre-elles, en géographie, concerne peut-être la manière d'étudier les pays dits « du Sud» qui reste aujourd'hui trop souvent ethnocentrée, mais on peut étendre ce raisonnement à la plupart des objets de la géographie contemporaine (le rural et l'urbain, le physique et l'humain, le politique et le culturel, la liste des oppositions binaires est longue, à leur rang paradoxalement il faut d'ailleurs aussi ranger le colonial et le postcolonial !). Car, certes, il est nécessaire de critiquer les postcoloniaux, mais il est bon aussi de ne pas oublier de critiquer aussi ce à quoi ils s'opposent. Certes on peut s'inquiéter des dérives d'analyses portant seulement sur les discours et les représentations (ou trop exclusivement «culturalistes » oubliant que le culturel ne peut être pensé hors de ses relations directes avec le politique en même temps qu'il est lié à des processus économiques) mais que dire de celles qui au contraire n'en tiennent aucun compte et négligent l'importance des imaginaires, des idéologies, des symboles? Certes aussi, "on a déjà donné ", mais peut-être faut-il donner encore, et ne pas négliger l'actualité des phrases de Frantz Fanon qui nous concernent directement en tant que géographes : «le contexte colonial (...) se caractérise par la dichotomie qu'il inflige au monde. La décolonisation unifie ce monde » (Les Damnés de la terre, édition de 2002, Paris, La Découverte, page 48), ceci à toutes les échelles et dans les représentations comme les faits matériels. 


\section{BIBLIOGRAPHIE}

Bayart Jean-François, 2010. Les études postcoloniales. Un carnaval académique. Editions Karthala, collection disputatio, $132 \mathrm{p}$.

\section{AUTEUR}

\section{PHILIPPE GERVAIS-LAMBONY}

Philippe Gervais-Lambony est Professeur des Universités, Université de Paris Ouest Nanterre gervais.lambony@wanadoo.fr 